\title{
La síntesis interna de la experiencia sensible. La psicología de Suárez y el origen de la neurociencia
}

\section{The Internal Synthesis of Sense Experience. Suárez's Psychology and the Origin of Neuroscience}

\section{JOSÉ ÁNGEL LOMBO}

Università della Santa Croce. Roma

lombo@pusc.it

Resumen. En el contexto de la gran síntesis filosófica elaborada por Francisco Suárez entre los siglos XVI y XVII, su comentario al De Anima manifiesta la originalidad de su pensamiento, entre la continuidad y la innovación ante las grandes cuestiones que la obra de Aristóteles dejaba abiertas. Su reflexión sobre los problemas del conocimiento abre el camino a un modo de pensar moderno, no exento de algunos aspectos problemáticos. Reconociendo una índole activa a las potencias sensibles y analizando también su dimensión fisiológica, reducirá la sensibilidad interna a una única potencia. La caracterización de este sentido interno único requiere una adecuada distinción respecto a la sensibilidad externa y respecto a la facultad intelectual, e implica una cierta localización cerebral. El diálogo de Suárez con la fisiología de su tiempo lo sitúa también en los inicios históricos de la neurociencia.

Palabras clave: Francisco Suárez; Aristóteles; teoría del conocimiento; sentidos internos; fisiología de los órganos sensibles; neurociencia. 
Abstract. In the context of the great philosophical synthesis of Francis Suárez, between $\mathrm{XVI}$ and XVII centuries, his commentary to De Anima manifests the originality of his thought, between continuity and innovation in front of the big issues that Aristotle's work had left open. His reflection on the problems of knowledge will open the path to a modern way of thinking, not exempt of some critical aspects. As he recognizes the active role of sensitive powers and analyzes their physiological dimension, he will reduce inner sensitivity to only one power. The characterization of this only inner sense requires an adequate distinction of it as regards both external sensitivity and intellectual power, and implies some localization in the brain. Surarez's dialogue with the physiology of his time places him at the historical beginning of neuroscience.

Keywords: Francis Suárez; Aristotle; theory of knowledge; inner senses; physiology of sensitive organs; neuroscience.

Hay muchas razones por las que Francisco Suárez merece ocupar un puesto relevante en la historia de la filosofía. Entre ellas, quizá pueda destacarse la peculiar encrucijada intelectual que le tocó vivir: la transición de la Escolástica Medieval al Pensamiento Moderno. Este período -de difícil delimitación, pero con características propias - es de una densidad que no deja de sorprender. La llamada "revolución científica”, la conexión entre el Viejo y el Nuevo Mundo, el crecimiento de las universidades a uno y otro lado del Atlántico, la consolidación de una cultura europea y la expansión de la Contrarreforma, son sólo algunos elementos de una época intelectual marcada por tensiones, pero también por un admirable desarrollo del saber. Es en este contexto en el que el Doctor Eximio desarrollará un pensamiento propio, fundado en la tradición, pero abierto a un modo nuevo - "moderno"de enfocar los grandes problemas de la teología y de la filosofía (Pereira 2007, 173-224). ${ }^{1}$

El objeto del presente trabajo es exponer el particular enfoque de Francisco Suárez acerca de los sentidos internos en su comentario al De

Sería prolijo, en este lugar, expandir el significado de esta incipiente "modernidad". En la generación sucesiva a Suárez, nos encontramos ya de lleno en la Filosofía Moderna, con autores en los que el Eximio ejerció un claro influjo: Descartes, Hobbes, Malebranche, Leibniz... Remitimos a la introducción de Castellote en la edición crítica: De Anima (Commentaria una cum quaestionibus in libros de Aristotelis De anima), S. Castellote (ed.), Sociedad de Estudios y Publicación, Labor, Madrid 1978-1991, pp. XXXVII-LXXIII. Citaré por esta edición. 
Anima de Aristóteles. La historia redaccional de esta obra es compleja, y la existencia de una edición crítica ha hecho nuestro trabajo mucho más asequible que de no haber existido ésta (Castellote 1978, XXXIX-XLVIII).

Como es sabido, Aristóteles recibe de Platón la idea de una división del alma de acuerdo con sus actividades. Sin embargo, mientras que para el Ateniense esto implicaba una separación entre varios principios vitales, el Estagirita asociará esas operaciones con diversas funciones de un mismo principio, manteniendo así la unidad sustancial del viviente. Estas funciones o facultades se encuentran, según él, jerárquicamente estructuradas en dos niveles: el sensible, con una base orgánica, y el intelectual, que es incorpóreo. A medio camino entre ellos, se destaca además un tercer grupo de potencias, orientadas a la actividad sensible, pero superiores a la sensibilidad externa. La tradición latina, árabe y judía los conocerá genéricamente como "sentidos internos".

La doctrina de Aristóteles acerca de estos sentidos no es enteramente sistemática, y pasará a la tradición posterior mediada por diferentes elaboraciones estoicas y neoplatónicas. Sin embargo, estas facultades reflejan paradigmáticamente la concepción aristotélica de la naturaleza humana como un todo corpóreo-espiritual; de aquí que su interpretación vaya a fluctuar, a lo largo del tiempo, entre la multiplicidad y la unidad, entre lo sensible externo y lo racional.

En la Edad Media, los sentidos internos alcanzaron una sistematización altamente concisa, tanto en el plano psicológico como en el fisiológico. En este contexto, destaca la reflexión de los filósofos árabes Avicena y Averroes. Apoyándose en ellos, Alberto Magno propondrá ya una clasificación estable de estos sentidos, con un fuerte énfasis en su dimensión corpórea. A través de él, su discípulo Tomás de Aquino toma contacto con los árabes y con Aristóteles, y, desde ellos elaborará su propia síntesis, que difiere en varios puntos de la de su maestro. En particular, el Aquinate dará un peso considerable a estas potencias en el proceso de la abstracción intelectual.

Las elaboraciones medievales -en ámbito árabe, hebraico y latinoofrecían, pues, una visión sistemática del papel de los sentidos internos en el proceso cognoscitivo; pero implicaban también una excesiva complejidad, 
que no parecía encontrar un claro reflejo en los textos aristotélicos y quizá tampoco en la experiencia. De aquí que, en el Renacimiento, fuera desarrollándose una tendencia a simplificarlos y a reducirlos en número. Así, en los comentarios al De Anima de la llamada "segunda escolástica", hacia la segunda mitad del s. XVI, encontramos ya la reducción de dichos sentidos a dos o a uno solo.

Francisco Suárez ocupa un puesto de gran relieve en este momento histórico, de manera particular en el contexto de los comentarios a Aristóteles en las universidades de Coimbra y Évora entre los siglos XVI y XVII (conocidos como “Cursos Conimbricenses”). Suárez reducirá los cuatro sentidos internos de Tomás a uno solo, aduciendo que una y la misma facultad puede aprehender y conservar, y puede hacerlo tanto respecto a las especies sensibles externas cuanto respecto a los valores prácticos elaborados internamente.

\section{El De Anima y la tradición aristotélica}

Como hemos apuntado, Aristóteles acepta la división de actividades del ser humano establecida por Platón (plano vegetativo, sensible e intelectual); pero, a diferencia de él, no considera que correspondan a tres almas, sino a tres tipos de actividad de una sola alma. Esto es coherente con su visión hilemórfica de la sustancia (unitaria y compuesta). Como queda patente en el De Anima, los sentidos están estructurados “hilemórficamente”, esto es, cabe distinguir en ellos un aspecto formal (la potencia operativa con su actividad inmanente) y otro material (el órgano corpóreo con su alteración física), pero siempre formando una unidad. Varios siglos más tarde, el médico Galeno se apoyaría en esa visión para elaborar una fisiología de las actividades del cuerpo en correspondencia con las del alma.

A partir de su visión del alma como dotada de distintas potencias, el Estagirita propone una psicología de las facultades mucho más elaborada que la de su maestro. Esta doctrina se encuentra sobre todo en el De Anima, pero debe entenderse también a la luz de otros escritos, como el De Memoria et reminiscentia y otros de los Parva naturalia. 
Nuestro punto de partida son precisamente las reflexiones del De Anima. En esta obra, tras haber expuesto las doctrinas de otros filósofos sobre el principio vital (libro primero) y haber ofrecido su personal definición de éste, el Estagirita examina sus distintas facultades, comenzando por las vegetativas y continuando con las sensibles (libro segundo). En el libro tercero, el Filósofo sube de nivel - por así decir-y centra su atención en la sensibilidad común, la imaginación y el intelecto. Concluirá con un estudio de la facultad motriz y la facultad desiderativa. Encontramos, pues, escasas referencias a la estimativa y a la memoria, que son examinadas con más detención en su tratado De memoria et reminiscentia.

El estudio de los sentidos internos tiene lugar, pues, en el libro tercero, en el contexto de explicar el conocimiento de una manera asociativa o sintética, respecto al carácter sectorial y analítico de los sentidos externos. Hay aquí, al menos, tres elementos oscuros si lo comparamos con la tradición posterior que nace precisamente de ese texto.

1 - El primero y más evidente es que no existe un término general para referirse a lo que la tradición posterior llamará "sentidos internos".

2 - En segundo lugar, Aristóteles habla del sentido común y de la fantasía, pero no hay una clara mención de la estimativa y de la memoria (los estudiará en el De memoria et reminiscentia).

3 - En tercer lugar, las referencias a la base orgánica de estos sentidos son nulas o muy vagas.

Por este motivo, la tradición posterior elaborará las posiciones de Aristóteles en diversas líneas, a través de desarrollos estoicos y neoplatónicos.

Quizá una contribución particularmente notable, en esta tradición, sea la del médico Galeno, de raigambre aristotélica, pero insertado en una época de predominio neoplatónico y estoico. Este autor conectará la doctrina estoica del conocimiento con elementos de la medicina de su tiempo, y propondrá no sólo una cierta división de los sentidos internos, sino también una precisa fisiología, localizada por él ya en el cerebro. Su propuesta gozará de gran prestigio en la Edad Media y en el Renacimiento, y lo veremos abundantemente citado por Suárez. 
Galeno asigna tres facultades cognoscitivas a tres partes del cerebro: la fantasía (ventrículo anterior); la razón particular o dianoetikón (ventrículo central) y la memoria (ventrículo posterior). Algunas conexiones desde el ventrículo anterior permitirían a los espíritus o humores llenar los nervios sensibles y motores, y así conectar los órganos sensibles y los músculos con el cerebro (Kemp-Fletcher 1993, 560).

Algo más de un siglo después, el cristiano Nemesio de Emesa seguirá esta teoría al asignar los distintos sentidos internos a ventrículos cerebrales.

En torno a este mismo período, en el mundo latino destaca San Agustín, quien habla de un "sentido interior" a medio camino entre la razón y los sentidos externos. Le concederá un valor marcadamente práctico y "propioceptivo”, pero sin llevar a cabo un particular análisis de su fisiología (De Civitate Dei, XI, 27; De libero arbitrio, II, 8; II, 12; Byers 2016, 57-59).

Las teorías medievales se desarrollarán desde estas premisas. En opinión de algunos estudiosos (Piro 2012, 182; Wolfson 1935, 71-73), la doctrina de los varios sentidos internos nació de la combinación entre el "sentido común” aristotélico, la topografía galénica de las facultades cerebrales y, más tarde, el añadido de la estimativa, ésta última conectada con lo que Aristóteles llamaba “opinión”, doxa (De Anima, III 3, 428 a 18-24). Estas teorías medievales tempranas seguirán tres líneas fundamentales: latina, árabe y judía. Entre ellas, muy probablemente las elaboraciones más sistemáticas tuvieron lugar en el seno de la filosofía árabe. Los filósofos árabes, en efecto, reciben a Aristóteles con la sistematización estoica y neoplatónica, probablemente a través de Juan Damasceno (Wolfson 1935, 80).

El filósofo y médico persa Avicena acoge ambas tradiciones - la filosófica de Aristóteles y la fisiológica de Galeno-y elabora su propia síntesis. Ésta tendrá un enorme peso en la Edad Media, especialmente en Tomás de Aquino, y se caracteriza por su carácter sistemático y pormenorizado. Puede decirse que la doctrina sobre los sentidos internos que circulará en el alto medioevo cristiano es esencialmente la elaborada por Avicena, considerada como una interpretación válida de la mente de Aristóteles.

Avicena propone la teoría más compleja y sistemática que encontramos antes del siglo XIII. Para él, los sentidos internos son al menos cinco (aunque, 
en ocasiones, habla de siete): el sentido común (que recoge las especies de los sentidos internos); la imaginación retentiva (que conserva las formas sensibles percibidas y las representa en los sueños o en el recuerdo); la cogitativa (entendida como imaginación compositiva, que acompaña a la elaboración intelectual); la estimativa (que permite a los animales captar los objetos sentidos como portadores de "intenciones no sentidas"2); y la memoria (que permite recordar las formas recibidas y las intenciones conectadas con ellas). Avicena vincula las mencionadas "intenciones no sentidas" con los movimientos afectivos. La reflexión sobre estas intenciones tendrá una particular relieve en la Edad Media y, de hecho, será uno de los puntos más criticados por Suárez respecto a los sentidos internos (Black 2000, 59-62).

Por su parte, Averroes continuará la teoría aviceniana sobre estos sentidos, pero distinguirá estas facultades en el hombre y en los animales. De aquí se seguirán dos importantes modificaciones. En primer lugar, considera superflua la facultad de la estimativa - tanto en los animales como en el hombre-, y, en su lugar, propone la cogitativa como facultad exclusivamente humana. Ésta reside en el cerebro y tiene la función de formular juicios sobre los singulares sensibles, función en la que se combina con el intelecto, dando lugar a la razón particular. Así pues, propone cuatro facultades sensibles internas: el sentido común, la fantasía-imaginación, la cogitativa y la memoria. De hecho, los animales no humanos sólo disponen de dos, esto es, sentido común e imaginación (Black 2000, 62).

En segundo lugar, Averroes acepta la existencia de "intenciones no sentidas”, pero las asocia ahora con la cogitativa y la memoria (en Avicena, era la estimativa, que Averroes considera cercana a la imaginación y, por tanto, superflua). Junto a esto, separa dichas intenciones de los movimientos

2 El concepto de “intenciones no sentidas" (intentiones insensatae) tendrá gran importancia en la reflexión medieval sobre los sentidos internos. En esta noción estriba la originalidad de la contribución árabe (particularmente de Avicena) a la caracterización de la estimativa-cogitativa (Black 2000, 70). En sentido propio, la expresión intentio insensata proviene de la Escuela de Salamanca (Joannes a St. Thoma 1937, III, p. 385, b. 14 [IV, q. 12, a, 1]). Sin embargo, podemos decir que el concepto estaba ya implícito en Aristóteles (De Anima III, De memoria et reminiscentia), fue formulado en la filosofía árabe (Avicena y Averroes), posteriormente elaborado en el pensamiento medieval y finalmente criticado por autores del Renacimiento y la primera modernidad, Suárez entre ellos (Wolfson, 1935). 
afectivos y, en cambio, les concede un papel específico en el conocimiento de lo singular (Black 2000, 62-63).

Tanto Avicena como Averroes tendrán gran influjo en Alberto Magno, particularmente el primero. Para el filósofo sajón, los sentidos internos son facultades orgánicas y cada uno de ellos tiene una localización precisa en el cerebro (Tellkamp 2012, 306-307). Por lo que se refiere al número y clasificación de estos sentidos, trata de ello en varias lugares - no sin ciertas fluctuaciones-, presentes sobre todo en dos obras: el De homine, qq. 35-43 (parte de la Summa de creaturis) y el Comentario al De Anima, separados entre ellos por casi quince años. En la primera de ellas, aparte del sentido común y de la memoria, considera la estimativa y la fantasía como análogas -en el plano sensible- al intelecto práctico y al especulativo (ST I, q. 39, a. 1, ad 1; Black 2000, 63). Esto significa, al menos, dos cosas: de una parte, en línea con Avicena, la estimativa es una facultad cognoscitiva práctica que mueve el apetito sensible; de otra, diversamente respecto a Avicena, depende del conocimiento previo de la fantasía (ST I, q. 39, a. 1; Black 2000, 64).

Posteriormente, en su Comentario al De Anima, considerará la imaginación no tanto como receptiva, sino también como retentiva. Esta imaginación además, combina las imágenes mediante la composición y la división, y recibe el nombre de "fantasía", que es la potencia sensible más alta (De Anima, II, 4, 7 y II, 1, 3). Por su parte, la estimativa tiene ahora el papel de aprehender las intenciones y de mover el apetito respecto a ellas, pero también de hacerse cargo de la individualidad de lo conocido, con cierta semejanza con la posición de Averroes (Black 2000, 64-65).

\section{La síntesis de Tomás de Aquino}

La doctrina aristotélico-árabe de la sensibilidad interna está presente en toda la psicología del Aquinate, quien la recibe inicialmente de Alberto Magno. Sin embargo, no encontramos muchos lugares en que cada uno de esos sentidos sea analizado pormenorizadamente y conectado con los otros. De hecho, los pasajes más importantes son dos, junto a otros más 
secundarios. Estos textos principales son Summa Theologiae I, q. 78, a. 4, y la Quaestio disputata de anima, a. $13 .^{3}$

De manera general, Tomás sigue las descripciones de Avicena, pero las corrige en momentos precisos con los enfoques de Averroes. Para establecer la diferenciación de los sentidos internos, su punto de referencia es la teleología de la naturaleza: «como quiera que la naturaleza no falla en lo necesario, es preciso que el alma sensitiva disponga de tantas operaciones cuantas sean requeridas para la vida del animal perfecto» (ST I, q. 78, a. 4, c.; también Quaestio disputata de anima, a. 13 c).

Los elementos más destacables de la sistematización del Aquinate nos parece que son los siguientes:

1 - Reconoce cuatro sentidos internos: sentido común, fantasía o imaginación, estimativa o cogitativa y memoria o reminiscencia.

2 - Señala que estos sentidos presentan diferencias entre el hombre y el animal, de aquí que algunos de ellos reciban nombres distintos: fantasía, estimativa y memoria, para los animales; imaginación, cogitativa y reminiscencia, para los seres humanos.

3 - Establece una distinción entre sentidos que captan y sentidos que conservan lo aprehendido.

4 - Distingue asimismo entre sentidos que perciben formas y sentidos que perciben valores prácticos (intentiones insensatae).

Respecto al punto 1, queda claro que el Aquinate lleva cabo una cierta síntesis entre Avicena y Averroes, muy probablemente inspirado por Alberto Magno. De otra parte, el punto 2 pone en evidencia lo que quizá sea uno de los elementos más originales de su análisis de los sentidos internos, a saber, la distinción de la facultad cogitativa respecto a la estimativa animal. En efecto, ésta última capta las intenciones prácticas no sentidas «sólo por un cierto instinto natural» (solum naturali quodam instinctu) (ST I, q. 78, a. 4, c.), mientras que aquélla lo hace «por medio de una cierta comparación» (per quandam collationem) (Ibidem), $\mathrm{y}$ «buscando y deliberando» (inquirendo et conferendo) (Quaestio disputata de anima, a. 13 c). De aquí que la cogitativa

3 Otros lugares con elementos relevantes, pero menos sistemáticos, son In II Sent., d. 24, q. 2 , a. 1, c, QDV, q. 25, a. 2, c y los comentarios al De Anima y al De memoria et reminiscentia. 
sea llamada también "razón particular” o “intelecto pasivo”, en cuanto es semejante a la razón universal, que compara conceptos universales (Ibídem).

En relación a los puntos 3 y 4, son con toda probabilidad los más críticos, como de hecho señalará el mismo Suárez, a saber: la distinción entre facultades receptivas y facultades retentivas (3), de una parte, y entre facultades representativas y facultades valorativas, de otra (4).

El Angélico mantiene, con Aristóteles (De Anima, II 6, 418 a 20-25), que los sentidos externos tienen no sólo objetos per se (propios o comunes), sino también per accidens: éstos son aquellos que, sin ser accesibles propiamente a dichos sentidos, son conocidos por ellos de manera indirecta, al conocer los sensibles per se (In Aristotelis librum De anima, II, 13, nn. 1-6). Estos objetos, pues, no son captados de manera propia y directa por los sentidos externos, sino por los internos. La facultad capaz de recibirlos es la estimativa-cogitativa, mientras que aquella que los conserva es la memoria-reminiscencia (In Aristotelis librum De anima, II, 13, n. 13). ${ }^{4}$

Se trata de objetos conocidos como intentiones (referencias de unas realidades a otras), que Tomás de Aquino describe como formas sensibles quas non percipit sensus exterior, no percibidas por las sensibilidad externa (ST, I, q. 78, a. 4, c). ${ }^{5}$ Aunque no las define con precisión, todo parece indicar que se trata de formas accidentales que caen bajo el accidente "relación" (con un significado semejante a habitudo) y no el de "cualidad" (como ocurre, en cambio, con los objetos per se de los sentidos externos). Además, el Aquinate señala claramente que la sensibilidad interna puede percibir estas formas secundum quod attingit rationem, en cuanto se encuentra conectada con la razón (In III Sent., d. 26, q. 1, a. 2, c; Allers 1941).

4 En este contexto, nos parece que los sensibilia per accidens no coinciden enteramente con las intentiones non sensatae, sino que son parte de ellas, siendo la otra parte objeto de la razón. Es decir, estas intentiones son captadas por la razón cuando son universales, y por la estimativa-cogitativa cuando son particulares. Seguimos a Jansen $(2005,53)$ y nos distanciamos en este punto de Fabro $(2008,158)$ quien identifica "sensibles per accidens" e “intenciones no sentidas". (De Haan 2014, 310-315).

5 En este contexto, el término intentio tiene un significado marcadamente relacional y práctico (referido al fin de alguna acción), mientras que species parece tener un significado más representativo-especulativo. En el ámbito de la sensibilidad interna, designan los objetos, respectivamente, del conocimiento "intencional" y del "formal" (Fabro 2008, capítulo IV). 
Una de estas intentiones, objeto de la estimativa-cogitativa, es el individuo conocido sensiblemente per accidens. Hay aquí una importante diferencia entre la estimativa animal y la cogitativa humana: ésta última conoce el individuo «como existente bajo una naturaleza común» (ut existens sub natura communi), mientras que aquélla lo hace «en cuanto es término o principio de alguna acción o pasión» (secundum quod est terminus aut principium alicuius actionis vel passionis) (In Aristotelis librum De anima, II, 13, n. 16). De aquí que la cogitativa juegue un papel fundamental en el razonamiento práctico, como indisolublemente unida a la razón (In IV Sententiarum, d. 50, q. 1, a. 3, ad s.c).

\section{Suárez, comentador del De Anima}

En 1572, cuando contaba con veinticinco años de edad, Francisco Suárez dio un curso de filosofía en Segovia que incluía un comentario al De Anima de Aristóteles. Aquel texto quedaría inédito hasta el final de su vida, ocurrida en 1617. En medio de ese tiempo, fue elaborando un programa de filosofía que correspondiera a su teología, fruto del cual serían sus Disputationes Metaphysicae. Quedaba pendiente, sin embargo, hacer lo propio con sus estudios de psicología. Por eso, en sus últimos años retomó el manuscrito de 1572 y buscó darle una forma definitiva. Sólo conseguiría hacerlo con los doce primeros capítulos del primer libro y algunas partes del capítulo sexto del segundo libro, de manera que el editor que los publicó de modo póstumo en 1621 -Baltasar Alvares - se encontraría fundamentalmente con dos versiones de una misma obra (Castellote 1978, XL).

Aristóteles y Tomás de Aquino son los autores principales que sirven de base a la Psicología del Doctor Eximio. Con todo, aunque seguirá su pensamiento en líneas generales, no dudará en separarse de ellos cuando lo crea conveniente, indicándolo en cada caso. En una tradición diferente, un punto de referencia importante también será el franciscano Duns Scoto. Finalmente, es notable su familiaridad con autores antiguos y medievales (Galeno, Avicena) y otros más cercanos a él, incluyendo la literatura médica (F. Vallés). Además, cabe señalar su actitud crítica ante Cayetano (Knuuttila 2014, 192-193). 
Por lo que se refiere a su teoría del conocimiento, Suárez la expone con cierta extensión en las disputationes 5-9 de su comentario al De Anima: las potencias cognoscitivas en general (5), los sentidos en general (6), los sentidos externos (7), los sentidos internos (8) y la potencia intelectiva (9). Uno de los principales problemas que se plantea en este contexto es si la sensibilidad es pasiva o activa. Rechaza que ésta sea puramente pasiva, pues eso implicaría que lo menos perfecto (la realidad física) causa lo más perfecto (el alma y su facultades). Se inclina así a conceder un carácter activo a las facultades sensibles que no aparecía en Aristóteles. ${ }^{6}$ En esta combinación de pasividad y actividad hay una "armonía” o "simpatía" entre los sentidos externos e internos, como también entre otros niveles de actividad (disp. VI, q. 2, n. 13; García Cuadrado 2015; Castellote 1963, 109-112). Para el Doctor Eximio, una potencia cognoscitiva informada por una especie constituye un instrumento integral, por medio del cual toda el alma obra (disp. V, q. 4, n. 16).

Suárez dedica al estudio de la sensibilidad interna la entera disputatio 8 de su Comentario al De Anima. Los pasajes a los que alude se encuentran en los capítulos 2 y 3 del libro III de la obra aristotélica.

Desde las primeras líneas, hay dos aspectos que merecen ser señalados: el autor pretende ofrecer su propia visión sobre los sentidos internos y, al mismo tiempo, proponer una válida interpretación de Aristóteles. Esto último puede parecer excesivamente ambicioso, sobre todo si tenemos en cuenta que su posición se distancia notablemente de otros intérpretes de gran autoridad, como Avicena o Tomás de Aquino. Para mantener la validez de su propia interpretación, Suárez se apoya indirectamente en la dificultad intrínseca de los textos y en su consiguiente ambigüedad. ${ }^{7}$

La Disputatio 8 tiene una breve introducción, en la que se describe la doctrina aristotélica sobre esta materia de un modo sucinto. Como es común

6 Se trata de la doctrina del "sentido agente", que había sido propuesta por Averroes y desarrollada antes que Suárez por Duns Scoto y Juan de Jandun, entre otros (Knuuttila 2014, 201-204).

7 Cfr. In De Anima, d. 8, proemio: "Haec docet Aristoteles de istis sensibus, et ea obscure et concise, quapropter maiore declaratione indiget haec materia. Breviter tamen de ea dicetur, quoniam pauciora nobis sunt nota de his sensibus". 
en las exposiciones del Eximio, el pensamiento aristotélico es presentado no de manera textual, sino esquemáticamente, condensado en cinco conclusiones de carácter asertivo:

1. Hay un sentido interior, distinto de los sentidos externos, capaz de conocer las operaciones y discernir entre los objetos de éstos.

2. Sentir es distinto de entender y de admirarse.

3. La fantasía se distingue de la estimativa.

4. La fantasía no es un sentido externo ni un hábito intelectual.

5. La fantasía es el movimiento producido por el sentido externo en acto.

A partir de aquí, se propone responder a dos preguntas, que corresponden a las dos quaestiones que componen la Disputatio: a) si hay un solo sentido interno o varios; y b) cuál es el objeto, operaciones, sujeto y órgano del sentido interno.

\section{a) Si hay un solo sentido interno o varios}

El hilo argumentativo del autor es muy pormenorizado. Su punto de partida es la existencia de la sensibilidad interna, caracterizada genéricamente como «potencia sensible distinta de los sentidos externos», ya se trate de una o de varias facultades. Para poder determinar su número, empleará un método analítico, esto es, pasará revista aisladamente a cada una de las modalidades de la sensibilidad interna, que muchos autores precedentes habían considerado como facultades distintas. Hablará así del sentido común, la fantasía, la imaginación, la estimativa, la cogitativa, la memoria y la reminiscencia.

En primer lugar, Suárez presenta el sentido común como una cierta facultad interior capaz de conocer los objetos de los sentidos externos en su conjunto, distinguiendo unos de otros. Es necesario al alma sensitiva principalmente para asociar objetos sensibles que no pueden caer bajo un mismo sentido externo (por ejemplo, un sonido y un color); pero también para tener noticia de las operaciones mismas de los sentidos externos. ${ }^{8}$

8 Esta segunda razón es presentada por Aristóteles. Otras razones aducidas por Alberto Magno, Tomás de Aquino o Avicena no son consideradas de suficiente peso por nuestro autor. 
La fantasía, por su parte, es descrita como el sentido interno capaz de conocer todas las realidades sensibles en ausencia de éstas, conservando las formas aprehendidas. ${ }^{9}$ Común a los hombres y a los animales irracionales -aunque no esté presente en todos-, era descrita por Aristóteles como «el movimiento hecho por el sentido en acto» (De Anima, III 3, 428 b 13). Según Suárez, esta expresión define el acto de la fantasía, pero no su índole propia como potencia; de aquí que prefiera una definición más analítica: «la potencia que recibe y conserva las especies de las realidades sensibles externas y obra por ellas en ausencia de los objetos». Tras la fantasía, Suárez continúa con la imaginación, y afirma que no es distinta de aquélla, sino que le añade la capacidad de componer los sensibles y de forjar cosas imposibles (Disp. VIII, q. 1, nn. 5-8).

Por lo que atañe a la estimativa, ésta es el sentido interior capaz de captar la realidad sensible según la razón de lo conveniente o lo inconveniente, y por ello puede mover y dirigir el apetito sensible. Aunque se trate de una operación común a todos los animales, este conocimiento práctico no es accesible a los sentidos externos (es insensatum). En el caso del ser humano, esta facultad no está guiada sólo por el instinto natural, sino por un mayor conocimiento y experiencia, e incluso por la misma razón, motivo por el cual recibe el nombre de "cogitativa” (Disp. VIII, q. 1, nn. 9-10).

En cuanto a la memoria, Aristóteles la describía genéricamente como aquella potencia sensible que conoce lo pasado en cuanto ya conocido. De manera más específica, algunos la comprenden como una cierta potencia capaz de conservar las intenciones, ya aprehendidas por la estimativa, en ausencia de los objetos (Disp. VIII, q. 1, n. 11).

Finalmente, la reminiscencia es la potencia que recuerda lo pasado, pero no de modo simple, sino con imaginación y con un cierto discurso. De aquí que muchos - Suárez entre ellos - no la consideren propiamente una facultad sensible, sino una actividad intelectual discursiva a la que acompaña una actividad sensible (Disp. VIII, q. 1, n. 12).

9 Se trata, por ello, de una facultad “abstractiva”, a diferencia de otras que pueden conocer sólo en presencia de la realidad, y que son llamadas “intuitivas". In De Anima, Disp. V, q. 1, n. 4. 
Desde estas premisas descriptivas, nuestro autor examina diferentes posiciones acerca del número de los sentidos internos. En un notable esfuerzo analítico, distingue las propuestas que establecen dos, tres, cuatro, cinco o seis facultades de esta índole. Los expondremos de manera esquemática por un motivo de claridad (Disp. VIII, q. 1, n. 13).

i) Dos sentidos internos:

a1- Sentido común / fantasía (que incluye la estimativa y la memoria): Suárez menciona aquí a Pérez, Turisano y Véneto.

a2- Sentido común-fantasía / estimativa-memoria: como partidario de esta opinión, nuestro autor cita a Marcelo.

ii) Tres sentidos internos: sentido común-fantasía / cogitativa / memoria: se trata de los teólogos Gregorio Niseno, Juan Damasceno y Nemesio de Emesa; y los médicos Galeno y Francisco Vallés.

iii) Cuatro sentidos internos: sentido común / fantasía / estimativa-cogitativa / memoria: ésta es, a decir de Suárez, la opinión más común, mantenida por Tomás de Aquino y por Averroes.

iv) Cinco sentidos internos: los cuatro mencionados más la imaginación, como potencia diferente: es la posición de Averroes, mantenida también por Alberto Magno.

v) Seis sentidos internos: los cinco apenas señalados más la reminiscencia, como facultad distinta: nuestro autor no señala un nombre particular en defensa de esta opinión. Probablemente, se trate de una visión integral de las posiciones de Avicena y Averroes (Lavin 1992, 301).

El paso siguiente de nuestro autor es la deconstrucción pormenorizada de estas opiniones. En general, las rechaza por carecer de suficiente fundamento, lo que ocurre cuando el criterio de distinción de las facultades no se basa en principios propios (los objetos y los actos), sino en principios diferentes (por ejemplo, aspectos accidentales de los objetos o la condición física de los órganos sensibles).

Dedica especial atención, sin embargo, a la posición de Tomás de Aquino y Averroes, por considerarla más probable que las otras. Ésta tiene, en efecto, un doble fundamento. De una parte, la distinción entre un conocimiento sensible por medio de especies sentidas (species sensatae) y otro por medio 
de especies no sentidas (species insensatae), que equivale a la distinción entre un conocimiento representativo (sentido común y fantasía-imaginación) y otro de tipo práctico (estimativa-cogitativa y memoria-reminiscencia), como antes apuntábamos. El segundo fundamento es la distinción entre un conocimiento sensible "en presencia del objeto" (intuitive), capaz de captar lo conocido, y otro "en ausencia del objeto" (abstractive), capaz de conservarlo. ${ }^{10}$ Ambas modalidades no pueden darse en una misma facultad orgánica - dirán Averroes y Tomás-, puesto que la aprehensión está facilitada por la humedad de los componentes del órgano, mientras que la retención lo está por su sequedad. Es la misma observación que hace Tomás de Aquino en ST I, q. 78, a. 4, c (e, indirectamente, en Quaestio disputata de anima, a. 13, c).

Para elaborar su propia posición, Suárez enuncia ante todo un principio: no deben multiplicarse los sentidos internos porque uno pueda realizar más actividades que otros (distinción de grado), sino porque uno no pueda realizar las actividades de otro (distinción de especie). Se trata, a mi modo de ver, de una síntesis del principio escotista-ockhamiano de parsimonia (numquam ponenda est pluralitas sine necessitate), y el principio aristotélico-tomasiano de que las formas más perfectas incluyen virtualmente las más imperfectas, y las potencias superiores incluyen virtualmente las potencias inferiores (De Anima, II 3, 414 b 28-31; Pseudo-Dionisio Areopagita, De divinis nominibus, V, 3 (PG III, 817); Tomás de Aquino, ST I, q. 76, aa. 3-4).

Una aplicación de los principios apenas mencionados es que no hay que poner en el hombre más sentidos internos que en el animal irracional, puesto que sus facultades superiores incluyen virtualmente las inferiores. Desde aquí, refutará a quienes sostienen cinco o seis sentidos internos, pues no hay, en el hombre, una real distinción entre fantasía e imaginación, entre estimativa y cogitativa, o entre memoria y reminiscencia.

10 La diferencia entre facultades “abstractivas” e “intuitivas” tiene sus raíces en Duns Scoto, pero es formulada explícitamente en los comentarios al De Anima de los maestros franciscanos Bartolomeo Mastri y Bonaventura Belluto. Será retomada por Suárez y la encontraremos también en Juan de Santo Tomás (Heider 2014, 235-237). 
Desde estas premisas, el Doctor Eximio ofrece su propia conclusión, que no será otra sino la reducción de todos los sentidos internos a uno solo. Para ello, seguirá dos pasos. De una parte, identificará las facultades que obran en presencia del objeto ("intuitivas") y aquellas que pueden hacerlo en su ausencia ("abstractivas"). De otra, establecerá una equivalencia entre las facultades que aprehenden formas y aquellas que captan intenciones (Disp. VIII, q. 1, nn. 17-21).

En primer lugar, apunta a la naturaleza receptiva de la potencia cognoscitiva. Ésta, en efecto, debe recibir una especie para poder conocerla. En los sentidos externos, sólo hay recepción de la especie en presencia de la realidad sensible, puesto que no pueden conservar la especie sin una inmutación por parte de la realidad física. Por su parte, la fantasía y la memoria son estimuladas por medio de la inmutación de los sentidos externos; por tanto, pueden conocer en presencia del objeto. La conclusión del autor es que no deben multiplicarse las potencias (como intuitivas y abstractivas) en razón de esos actos diferentes, pues pueden convenir en una misma facultad (Disp. VIII, q. 1, n. 17). ${ }^{11}$

Como confirmación de esta conclusión, el autor pretende disolver la justificación de Tomás de Aquino, a saber, que las potencias sensibles aprehensivas y las conservativas deban distinguirse en virtud de la disposición de sus órganos (que han de estar o húmedos o secos, respectivamente). En efecto, la inmutación del sentido externo (aprehensiva) y de la fantasía (conservativa), no pueden ser sucesivas, sino simultáneas; y, por tanto, deben darse unidas.

A esto añade que la imaginación puede asumir funciones del sentido común, a saber, atender y concurrir con todos los sentidos externos a sus

11 El razonamiento de Suárez es complejo, sobre todo por su uso de los verbos modales "poder" y “deber". Hemos pretendido reflejarlo en nuestra explicación, buscando al mismo tiempo la claridad. De otra parte, refuerza su conclusión con otra prueba («Patet ultima consequentia...») cuyo significado parece más bien oscuro. La frase de la edición crítica dice «potentia, quae potest immutari ab obiecto praesenti, potest attente immutari...», lo cual no parece coherente con el hilo lógico del párrafo. La traducción castellana no ayuda a entenderla, pues la elude parcialmente. El texto resultaría bastante más inteligible con la preposición absque, en lugar de $a b$; es decir, la frase podría quedar así: «potentia, quae potest immutari absque obiecto praesenti, potest attente immutari...». 
sensaciones. Señala además que la distinción, en el plano orgánico, entre lo receptivo y lo conservativo no es rígida, como demostraría el caso del plomo. Pero tampoco basta la disposición del órgano para causar la actividad de la potencia, ya que la especie no es recibida sólo en aquél, sino también en la facultad misma, en la medida en que es la propia naturaleza de ésta (ex cuis natura provenit) la que determina el modo de la recepción de la especie. Añade, de manera marginal, pero clara, una referencia a que tal distinción de disposiciones no se da en la parte del cerebro en la que se coloca el sentido interno. ${ }^{12}$

De todo esto, concluye que no deban distinguirse, entre las potencias internas, aquellas que conocen en presencia del objeto (aprehensivas) y las que lo hacen en su ausencia (conservativas). Por tanto, identificará el sentido común y la imaginación, de una parte, y la estimativa y la memoria, de otra (Disp. VIII, q. 1, n. 17).

En segundo lugar, nuestro autor se propone disolver también la distinción entre los sentidos internos representativos (sentido común-fantasía) y aquellos otros valorativos (estimativa-memoria). Ya antes, en esta misma obra, había liquidado la distinción entre especies sentidas (sensatae) y no-sentidas (insensatae) (Disp. VI, q. 2, n. 15). Centrándose ahora en las facultades, sostiene que una potencia que es capaz de conocer la realidad «bajo un aspecto no sentido» (sub ratione insensata), debe poder conocerla también «bajo un aspecto sentido» (sub ratione sensata) (disp. VIII, q. 1, n. 19). La razón de ello parece ser ante todo experiencial: el acto por el que una realidad es conocida en sus aspectos formales es el mismo por el que es conocida en su valor de conveniencia o inconveniencia («hic est inimicus»).

12 Esto último parece una referencia velada a Galeno, aunque quizá pueda ser también a Vallés. Cfr. Disp. VIII, q. 1, n. 18. No afirma aquí Suárez que la disposición del órgano sensible sea irrelevante a la recepción de la especie; pero sí parece considerarla secundaria respecto a la disposición de la facultad. Esa disposición no parece adquirida (tendría que ser o un hábito o la misma disposición física del órgano), sino natural; es decir, parece ser su propia naturaleza como «tal» facultad. Esto puede dar pie a una consideración de la facultad sensible como «separable» de su órgano, lo cual es altamente problemático. 
Un motivo que se añade al anterior es que la misma fantasía puede mover el apetito, como había señalado Aristóteles. ${ }^{13}$ Desde aquí, nuestro autor niega la distinción entre facultades sensibles formales e intencionales, y rechaza, en general, que un juicio especulativo y uno práctico requieran facultades diferentes (Disp. VIII, q. 1, n. 20). ${ }^{14}$

En consecuencia, Suárez ha identificado sentido común y fantasía, estimativa y memoria, y estimativa y fantasía. Por tanto, concluye, el sentido interno es uno solo realmente. La imaginación, la cogitativa y la reminiscencia son sólo distintos grados de perfección, en el hombre, de las potencias mencionadas (Disp. VIII, q. 1, n. 21).

Al término de esta cuestión, el Eximio tienen aún dos tareas: indicar la autoridad de otros autores que, en principio, apoyarían su propuesta; y salir al paso de algunas posibles críticas. Como autoridades, invocará a Aristóteles y a Tomás de Aquino. En primer lugar, el Estagirita se refiere en ocasiones a un único sentido interno, al que asigna como objeto los sensibles comunes, el recuerdo, la unificación de las sensaciones externas y la conservación de especies ya recibidas (Disp. VIII, q. 1, n. 21; Aristóteles, De memoria et reminiscentia, I, 450 a 9-13; De insomniis, I, 459 a 14-22). Además, en algún texto, el Filósofo identificaría explícitamente la fantasía y la memoria (De memoria et reminiscentia, I, 450 a $22-25) .{ }^{15}$

13 El texto aristotélico (De Anima III 9, 432 b 15 ss) es bien conocido y, por lo demás, es una afirmación que también encontramos en Tomás de Aquino (ST, I, q. 81, a. 3, ad 2). El propio Estagirita señala que, en ese lugar, se refiere genéricamente a la imaginación como sinónimo de intelecto. En otras ocasiones, distingue claramente entre "imaginar" y "opinar" como actividades respectivamente descriptiva y valorativa (De Anima, III 3, 427 b 23). De otra parte, no hay inconveniente en afirmar que el apetito sensitivo puede ser movido por la imaginación y los sentidos; pero tal movimiento no parece ser inmediato, sino remoto, esto es, a través de la estimativa (Tomás de Aquino, In II Sent., d. 24, q. 2, a. 1, ad 2).

14 Esta última afirmación sorprende, porque concierne claramente a la facultad racional, pero no parece que pueda aplicarse a los sentidos. En efecto, la unidad de la razón no proviene de la indiferencia de sus actos (especulativos y prácticos), sino de su propia naturaleza inmaterial, y esto no puede decirse de los sentidos.

15 Sin embargo, el Estagirita no parece identificar ambas facultades, sino sólo indicar que se dan siempre juntas. 
Por lo que se refiere al Aquinate, nuestro autor es más cauto («insinuat $D$. Thomas...») y cita un texto relativo a los sueños (ST, I, q. 84, a. 8, ad 2), en el que el Angélico afirma que el sentido común puede activarse durante el sueño -en ausencia del objeto-, mientras que sostiene en otro lugar (ST, I-II, q. 15, a. 1, c, y q. 17, a. 7, ad 3) que dicha facultad sólo puede ser inmutada en presencia del objeto. Estos últimos textos de Tomás de Aquino, sin embargo, no se refieren al sentido común, sino a la sensibilidad externa.

Como posibles críticas a su planteamiento, Suárez apunta a dos: la constatación de una multiplicidad de potencias sensibles en virtud de su índole corpórea (lo que debería afectar también a los sentidos internos) y el hecho de que su postura no sea prácticamente compartida por nadie (sic). El autor acepta la primera afirmación en relación a los sentidos externos, pero no para el sentido interno, puesto que se trata de una potencia universal en el nivel sensible. En cambio, si la comparamos con la universalidad del intelecto, aparece como limitada y determinada a un objeto más particular. Respecto a la segunda crítica, en cambio, considera que no hay ninguna parte de su conclusión que no sea aceptada por muchos autores. Es más, afirma, tiene a su favor la autoridad de Aristóteles (Disp. VIII, q. 1, n. 22).

Finalmente, y a modo de cierre de esta primera cuestión, nuestro autor se pregunta si los sentidos internos pueden distinguirse al menos "formalmente" (saltem formaliter), puesto que ha probado que son realmente distintos (Disp. VIII, q. 1, n. 2). ${ }^{16}$ La respuesta parecería afirmativa, pues las definiciones de los mencionados sentidos son diferentes; sin embargo, nuestro autor lo rechaza, pues esas diferencias no indican un objeto formal distinto en las potencias. Se trataría de algo análogo a lo que sucede con la facultad intelectual, que recibe muchos nombres (intelecto, razón, memoria, intelecto práctico, especulativo, etc.), siendo real y formalmente una sola (Disp. VIII, q. 1, n. 24). Por lo tanto, el sentido interno es real y formalmente uno solo, y toda distinción en él es sólo de razón.

16 Esta alusión a una posible “distinción formal” es una referencia a Duns Scoto, quien había introducido esta distinción entre la "real” y la "de razón” para significar aquella que existe entre varias formalidades o aspectos de una misma realidad. Por su parte Suárez propone una “distinción modal” en el objeto del sentido interno (Beuchot 1994). 
b) Objeto, operaciones, sujeto y órgano del sentido interno

Para Suárez, los razonamientos anteriores, han establecido "la verdad sobre el sentido interno" (Disp. VIII, q. 2, n. 1), pues, al demostrar su carácter único, se han puesto las bases para entender su naturaleza. A continuación, y sobre este fundamento, es fácil determinar todos los elementos que le corresponden (Ibidem).

En primer lugar, indica que su objeto es "todo lo sensible percibido por el sentido externo”. En efecto, el sentido externo es -como ya ha apuntadouna potencia universalísima en su grado, por lo que le corresponde un objeto asimismo universalísimo en ese grado, a saber, "todo lo sensible" (Ibidem).

Junto a esto, nuestro autor hace otra interesante observación. En los sentidos externos, hay unos objetos propios de cada uno y otros comunes a varios, que son ciertas modalidades de los anteriores. De manera análoga, en el objeto del sentido interno, hay un objeto propio y una serie de modalidades, con una jerarquía entre ellas (Ibidem). ${ }^{17}$ En primer lugar, el objeto propio del sentido interno, accesible por su propia especie, es "lo sensible en general" (todo lo sensible). En segundo lugar, de forma derivada y como una modificación de su objeto propio, es capaz de conocer las "razones no sentidas" (rationes non sensatae), esto es, los aspectos de conveniencia o nocividad, más las operaciones de los sentidos externos. Por último, puede conocer también todos aquellos aspectos que no son representados por las especies de los sentidos externos y que, sin embargo, le son accesibles. ${ }^{18}$

Por lo que se refiere a los actos propios del sentido interno (ya que deben ser varios), nuestro autor se expresa con precisión, con una fórmula en la que incluye exhaustivamente todas las modalidades operativas señaladas. Los actos de este sentido son "el conocimiento de todas las realidades sensibles,

17 Vemos aquí que Suárez ha modificado notablemente el enfoque de Averrores y Tomás de Aquino sobre las funciones del sentido común (por ejemplo, Tomás de Aquino, Sentencia De anima, lib. 2, 1. 13) y también de la estimativa. Sobre las “modalidades" del objeto de conocimiento, cfr. Alarcón 2014.

18 Esta última modalidad resulta algo vaga, pero se entiende si no perdemos de vista que está hablando del objeto de una facultad interna única con un objeto universal en su grado. Por el contexto, esta última modalidad podría corresponder a los sensibilia per accidens (aunque ha negado antes que puedan inmutar el sentido: Disp. VI, prooem., n. 1). 
la distinción entre ellas, el juicio práctico y especulativo sobre la realidad y la memoria de las cosas pasadas” (Disp. VIII, q. 2, n. 2).

A continuación trata del sujeto del sentido interno. Por sujeto entiende aquí nuestro autor el tipo de individuo viviente que posee una determinada facultad. Por ello, preguntarse por el sujeto del sentido interno equivale a dilucidar qué animales están dotados de esta potencia. En general, Suárez sostiene que está presente en todos ellos, puesto que todos tienen apetito, y ambas facultades se dan unidas. ${ }^{19}$

Sin embargo, este sentido no se da en el mismo grado de perfección en todos los animales ni pueden todos ellos realizar las mismas operaciones. En primer lugar, aquellos que sólo disponen del sentido externo del tacto -o también del gusto-, tienen un sentido interno imperfectísimo, que no es capaz siquiera de conservar las especies en ausencia del objeto (Disp. VIII, q. 2, n. 3). ${ }^{20}$ Un grado algo superior corresponde a los animales que, aun disponiendo de varios sentidos externos, tampoco pueden conocer en ausencia del objeto; pero en ellos la sensibilidad alcanza un ámbito mayor que los antes mencionados (es, en cierta medida, "más universal”). Los animales perfectos, en fin, tienen un sentido interno asimismo perfecto, el cual incluye las "modalidades" de la memoria (ratio memoriae), de la estimativa (ratio aestimativae) y del sentido común (ratio sensus communis); y, por ello, tienen también "prudencia natural” y "capacidad de aprendizaje” (disciplinabilitas) (Disp. VIII, q. 2, n. 4). ${ }^{21}$

Entre estos animales perfectos, se encuentran, a decir de nuestro autor, no sólo aquellos complejos cuyos sentidos externos están claramente desarrollados (como los elefantes), sino también algunos otros más simples,

19 El apetito, en efecto, requiere un juicio práctico sobre la conveniencia o nocividad de un objeto. En este sentido, Aristóteles afirmaba que todos los animales tienen fantasía (cfr. De Anima III 10, 433 b 28).

20 Ya Aristóteles había señalado la posibilidad de un animal con sólo el sentido del tacto (De Anima II 2, 413 b 8).

21 La mención de la ratio memoriae, ratio aestimativae y ratio sensus communis es una prueba explícita de una teoría del conocimiento de tipo "modal”. En este contexto, la diferencia entre "modalidades" del sentido interno se acerca bastante a la distinción de facultades, como la encontramos en otros autores, para los que la "sensibilidad interna" podría ser considerada una suerte de género con distintas especies. 
como la hormiga y la abeja, los cuales tienen memoria (dedicará un cierto espacio a demostrarlo) y, por ello, "prudencia natural". Si la memoria se da, además, con el oído, nos encontramos con animales que pueden ser domesticados (disciplinabilia). (Disp. VIII, q. 2, n. 5). ${ }^{22}$

Estas observaciones muestran la importancia que Suárez concede a los "modos" del conocimiento. En efecto, afirmará que "el sentido interior conoce las sensaciones de los sentidos externos no como su objeto propio, sino como modos de su propio objeto" (Disp. VIII, q. 2, n. 5). De aquí que el objeto conocido por el sentido interior pueda perfeccionarse según el modo de la memoria (el fantasma pasa a ser recuerdo de la experiencia ya tenida), como también puede disminuir y corromperse (puede perder aspectos circunstanciales que componen dicho objeto: por ejemplo, olvidando detalles).

Especial interés histórico revisten las reflexiones del Eximio sobre el órgano del sentido interno. En efecto, señala que Aristóteles, al asignar la raíz de la actividad sensible al corazón, negó “consiguientemente” (sic) que este sentido tuviera un órgano (De iuventute et senectute, III, 469 a $5 \mathrm{ss}$ ). ${ }^{23}$ En cambio, él mismo afirmará "consiguientemente" que este órgano se encuentra en el cerebro. A favor de esta posición estarían Galeno, Hipócrates y otros (Disp. VIII, q. 2, n. 7). ${ }^{24}$

Para mostrar esta relación de consecuencia, nuestro autor empleará varios argumentos tomados de la experiencia (Disp. VIII, q. 2, n. 7$)^{25}$. En primer lugar, ofrece un motivo que arranca de una convicción fenomenológica

${ }^{22}$ El autor indica que las abejas están probablemente dotadas de oído y, por tanto, pueden -en sentido amplio- aprender: Ibídem, n. 6.

23 Hemos escrito el adverbio "consiguientemente" (consequenter) entre comillas para llamar la atención sobre un hecho particular: no se entiende qué relación de consecuencia existe entre afirmar la centralidad del corazón como órgano principal de la vida sensitiva (y vegetativa), y negar que el sentido interno tenga un órgano. Por tanto, el consequenter de Suárez parecería que queda sin resolver. Una posible explicación me la ofreció el profesor Heider: si el órgano de la sensibilidad interna es el cerebro y éste es un órgano unitario, esto comporta también la unicidad de la facultad. Es decir, la "relación de consecuencia" (consequenter) se daría entre la unicidad del sentido interno como facultad y la unicidad del cerebro como órgano.

24 La oposición entre Aristóteles y Galeno sobre la centralidad, respectivamente, del corazón y del cerebro, es tema clásico en la historia de la anatomía (Blanco 2014, 29 ss).

25 Con la expresión experientia parece aludir nuestro autor no a la experiencia común, sino a los datos empíricos de la práctica y la reflexión médicas. 
inmediata, a saber, la de experimentar subjetivamente que la imaginación y la fantasía están en la cabeza (por ejemplo, ante la experiencia del "cansancio de cabeza”) y el dato anatomo-patológico de que "la lesión de los sentidos proviene de la lesión del cerebro" (Disp. VIII, q. 2, n. 7). Otro motivo es la opinión común de que los sueños, producidos por la imaginación, tienen lugar en la cabeza (Ibídem).

A continuación, se pregunta en qué parte del cerebro se encuentra el sentido interno. Con la anatomía de su tiempo, responderá que el cerebro está dividido en tres cavidades: anterior, media y posterior. A ellas, Galeno había asignado la base orgánica de tres sentidos internos, respectivamente: sentido común-fantasía, cogitativa y memoria. Las lesiones de esas zonas provocarían un mal funcionamiento de los sentidos correspondientes, hasta provocar la demencia. Ésta tendría, por tanto, tres posibles causas. En primer lugar, la lesión de la parte anterior, ${ }^{26}$ que impide la actividad del sentido común y, con ella, de los sentidos externos, que derivan de él. En segundo lugar, el deterioro de la cavidad media, que indispone la actividad del juicio o de la razón. En tercer lugar, el daño de la parte posterior, que comporta el trastorno de la memoria. Si se lesionaran las tres partes en su conjunto, tendríamos un trastorno general de todas las operaciones, como sucede en los “frenéticos" (Disp. VIII, q. 2, n. 8) ${ }^{27}$.

Esta descripción, sin embargo, no es rígida ni absoluta, ya que el sentido interior no puede estar dividido y, además, el mismo Galeno ofrece interpretaciones distintas en varios lugares. De modo sutil, nuestro autor apunta que tal vez la cavidad posterior sea el órgano del sentido interno, como parece sostener Vesalio (De humani corporis fabrica, VII, 6).

Para concluir, nuestro autor se plantea y resuelve dos dudas. De una parte, se pregunta si puede existir una lesión en el sentido interno sin que se dañe el externo; ${ }^{28}$ de otra, si la imaginación puede provocar de suyo un

26 El texto suareciano dice aquí «interior»; pero el contexto induce a pensar que se trata de la parte anterior.

27 Con el término phrenetici, probablemente se está refiriendo a lo que hoy entendemos por esquizofrénicos.

28 Aquí el texto dice “interno”, pero el contexto y la lógica del párrafo requiere que se trate de "externo". Así lo refleja la traducción en la edición de Castellote. 
daño, como ordinariamente se piensa. A la primera duda, responde que, en efecto, puede haber un mal funcionamiento del sentido interno y quedar intactos los órganos de los sentidos externos (quien padece una enfermedad mental, por ejemplo, puede tener la vista o el oído sanos). La razón de ello es que la capacidad de sentir proviene del sentido interno y se deriva, desde él, a los sentidos externos. De aquí que el sentido interno requiera, citando a Vallés, una disposición adecuada propia, que no proviene de los sentidos externos. A la segunda duda, responde también afirmativamente, pues la operación imaginativa mueve los "espíritus animales" y los difunde por todo el cuerpo (Disp. VIII, q. 2, n. 9).

\section{En los albores de la neurociencia}

El Comentario suareciano al De Anima pone de manifiesto la inspiración aristotélica -y, en buena medida, también tomasiana- de la Psicología del Doctor Eximio. Esta inspiración, como hemos visto, es bastante flexible y se encuentra frecuentemente matizada por otras líneas de pensamiento, en el conjunto de su vasta cultura y erudición. El aristotelismo suareciano, sin embargo, se descubre no sólo en el marco conceptual propio del Estagirita, sino también en su enfoque característico, a saber, la concepción del ser humano como una realidad unitaria espiritual-corpórea que actúa de acuerdo con esa naturaleza. Esta visión había sido precisada y transformada a lo largo del tiempo, pero - con sus múltiples variaciones- llegaba hasta Suárez a través del canal de autores que combinaban la medicina y la filosofía.

No podemos soslayar un elemento que puede parecer un límite, pero que no deja de ser profundamente significativo: el De Anima fue concebido por su autor como un tratado de filosofía natural, dentro del conjunto de los Parva naturalia ${ }^{29}$. De aquí que toda consideración de esta obra deba rendir

29 No es posible que nos detengamos ahora en considerar la continuidad y discontinuidad entre esta obra y la Ética a Nicómaco; pero es claro que entre ambos escritos -con sus netas diferencias- existe una profunda conexión de fondo: el ser humano es concebido como una unidad espiritual-corpórea, que tiende a su propio fin por medio del ejercicio del logos y de su propia corporeidad, en relación con otros seres racionales y con el mundo físico. 
cuenta de la continuidad entre los aspectos corpóreos y los estrictamente racionales del ser humano. Esto se hace particularmente evidente en el caso de la sensibilidad interna, y las reflexiones de Suárez lo ponen claramente de relieve: las facultades sensibles forman una unidad con sus órganos, y existe además una continuidad entre los sentidos y la razón (García Cuadrado 20015). De aquí la importancia que concede el Eximio a las disposiciones orgánicas en relación con las actividades del alma, sin que esto le lleve a confundir los actos inmanentes con los transitivos, la psicología con la fisiología.

En la tradición aristotélico-galénica, es notable la preocupación -y los hallazgos- acerca de la fisiología de los órganos de los sentidos. Ya hemos señalado las numerosas ocasiones en que aparecen, en el comentario suareciano al De Anima, citas de Galeno, Andrea Vesalio y Francisco Vallés. En particular éste último -discípulo de Vesalio y sucesor suyo como médico de Felipe II- era coetáneo de Suárez, y llegó a establecerse entre ellos un influjo recíproco. Vallés fue con gran probabilidad el canal por el que el Eximio conoció el pensamiento y la obra de Galeno (Madeira 2009).

Galeno siguió en muchos aspectos a Aristóteles, pero se distanció de él en una posición fundamental: mientras el Estagirita ponía en el corazón el centro de la sensación y el origen de los movimientos, Galeno situaba estas importantes funciones ya en el cerebro. Sin embargo, éste último consideraba los ventrículos cerebrales de manera semejante a los del corazón, de manera que los nervios serían conductos de los humores del cerebro y la médula (spiritus animalis) hacia todo el organismo, lo que en último término explicaría las sensaciones y las emociones.

Andrea Vesalio supuso un punto de inflexión en este proceso. En 1543, publicó el libro De humani corporis fabrica, a partir de un vasto conocimiento de la anatomía humana adquirido en el estudio y en la práctica quirúrgica. En él, sirviéndose de la ayuda de grandes ilustradores de la época, transmite de manera gráfica y pormenorizada un saber que había permanecido inaccesible hasta ese momento ${ }^{30}$. Concretamente, dedicará un entero capítulo al

30 En otro lugar hemos señalado que «a pesar de su indudable utilidad, el problema de este modo de concebir el cuerpo humano es que abre fácilmente a una visión mecanicista, según la cual el todo se explica por el análisis de sus partes y es resultado o efecto de éstas»: 
sistema nervioso y otro al cerebro, corrigiendo en buena medida el concepto galénico sobre la conducción de los humores por medio de los nervios al resto del organismo. Precisará asimismo la disposición de los ventrículos cerebrales propuesta por Galeno, que había sido comúnmente aceptada en la Edad Media (Vesalio 1543, capítulos IV y VII).

A pesar de las correcciones de Vesalio, la teoría galénica de localización ventricular de las funciones cerebrales continuaría siendo la más aceptada, y así la mantuvo también Vallés. Este autor acepta la división de Galeno de tres sentidos internos, pero les asigna cinco actividades diferentes (Vallés 1556, 70). ${ }^{31}$ En esta línea, propone su doctrina de la simpatía o armonía natural de las facultades, que tendrá una notable influencia sobre Suárez (García Cuadrado 2015).

\section{Visión de conjunto y observaciones críticas}

No puede negarse al Doctor Eximio un vasto conocimiento de los enfoques de otros autores y la originalidad de su propuesta. Manteniendo una propia coherencia - en buena medida, aunque no exclusivamente, basada en Aristóteles y Tomás de Aquino-, lleva a cabo un análisis de los problemas más acuciantes relativos al conocimiento sensible y propone una síntesis entre sus posibles soluciones. Es verdad que, alguna vez, ha sido etiquetado de ecléctico (Mahieu 1925), pero lo ha sido casi siempre en un sentido positivo (Grabmann 2006, 169). Suárez no busca un equilibrio entre opiniones diversas, sino que presenta sus propuestas como una opción superadora, frecuentemente original. No es fácil decir si se mantiene en la tradición más de lo que se abre a lo novedoso.

Su punto de partida es común a todos los tratados sobre el De Anima: la ambigüedad del Estagirita en torno al problema de los sentidos internos

J. A. Lombo - J. M. Giménez Amaya, Biología y racionalidad: El carácter distintivo del cuerpo humano, EUNSA, Pamplona 2016, p. 37.

31 Algunos autores sostienen que la localización cerebral de los tres sentidos (cogitativa, razón y memória) no proviene de Galeno, sino de Nemesio de Emesa (Barbado 1946, I, 437-438). 
y la necesidad de definir su estatuto gnoseológico. Su método, en cambio, es bastante personal: no es ya propiamente un comentario, entendido como una glosa del texto original ${ }^{32}$ sino una discusión continua de éste desde una permanente confrontación de perspectivas. En este sentido, el Eximio conoce ampliamente la tradición aristotélica, particularmente de los árabes (Avicena, Averroes); pero demuestra también una gran familiaridad con la fisiología antigua (Hipócrates, Galeno) y moderna (Vesalio, Vallés). Su propia propuesta sale al paso así de una serie de problemas que no habían quedado completamente resueltos en esa tradición, a saber:

De una parte, la distinción y continuidad entre el conocimiento intelectual y el sensible, que comporta, en éste último, la unidad entre las facultades y sus órganos.

En segundo lugar, la necesidad de postular un nivel interno de la sensibilidad, no reducible a las facultades externas; pero, en cualquier caso, distinto también del conocimiento intelectual.

Finalmente, la caracterización de la sensibilidad interna de acuerdo con dos criterios fundamentales: la distinción entre la recepción y la conservación del objeto sensible, y la divergencia entre la aprehensión de formas y la captación de intenciones prácticas.

La solución propuesta por Suárez, como hemos mostrado, es sintética, pero no ecléctica. Afirmará que el sentido interno es uno solo y que sus operaciones son, en realidad, "modalidades" del procesamiento interno de la experiencia sensible. Así, el sentido interno abarca todas las realidades sensibles, haciéndose cargo de ellas en cuanto inmediatas o ausentes, en cuanto presentes o pretéritas, de modo especulativo o de modo práctico. El enfoque de fondo de esta síntesis, más que de una simplificación, parece tratarse de una unificación de la experiencia.

Ya hemos apuntado que el comentario del Doctor Eximio al De Anima es una obra compleja, redactada al inicio y al final de su carrera docente, y de hecho inacabada. De aquí que contenga, en mi opinión, sólidas contribucio-

32 Ni siquiera incluye una traducción del texto original, y el esquema de su tratado sólo vagamente refleja el de la obra del Estagirita; se trata más bien de un tratado independiente sobre el alma (Simmons 1999). 
nes, pero también algunos elementos discutibles e incluso problemáticos. Sin pretender ser exhaustivo ni poder elaborarlos enteramente en este lugar, me limitaré a apuntarlos brevemente.

Respecto a Aristóteles que consideraba los sentidos como "potencias pasivas” (De Anima, II, 5), el Eximio tiene una concepción “activa” de la sensibilidad, pues considera que sólo desde esa posición puede justificarse el acto mismo de conocer en el plano sensible. Desde aquí, y por más que subraye la unidad entre la potencia y su órgano -como también entre todas las facultades en el alma-, lo cierto es que hay siempre una tensión entre la pasividad del órgano -que puede ser influido causalmente- y la actividad de la facultad sensible -que deriva del alma misma (Disp. V, q. 4, n. 16; South 2001, 237-238).

En el plano sensible interno, la pluralidad de niveles de actividad no implica, para Suárez, una diferenciación de facultades, sino una multiplicidad de "modalidades" operativas de un único sentido (disp. VIII, q. 2, n. 4). Nuestro autor parece conceder así a la sensibilidad interna algunas prerrogativas que Tomás de Aquino había asignado al intelecto: el poder recibir y conservar las especies, o el percibir su dimensión especulativa y práctica, permaneciendo una y la misma facultad, sin que la diversa disposición física de los órganos comporte la distinción de varias facultades orgánicas.

El propio Doctor Eximio reconoce que esta unidad-unicidad del sentido interno suscita una petición de principio, en cuanto se trata de una facultad orgánica; y lo resuelve desde la universalidad de esta potencia sensible en su propio orden (Disp. VIII, q. 1, nn. 21-22). De esta suerte, sin embargo, la capacidad operativa del sentido interno parece separarse de las disposiciones físicas de su órgano, casi como si dicha facultad fuera espiritual o que su unión con su base orgánica no fuera intrínseca. ${ }^{33}$ Se trata, sin duda, de aspectos que requerirían un estudio más detallado y una visión de conjunto de la obra del Doctor Eximio. Por el momento, nos limitamos a apuntarlos.

33 Recordemos que, para Tomás de Aquino, la facultad sensible es siempre forma de un órgano y, como tal, constituye una unidad con él, no sólo desde el punto de vista operativo, sino también en su propia estructura constitutiva. In De Anima, II, 2, 24, n. 5; ST, I, q. 78, a. 4 , c; $S T$, I, q. 85 , a. 1 , c. 


\section{Referencias}

Alarcón, E. 2014. “La dimensión modal del conocimiento.” Espíritu 63/148: 251-282.

Allers, R. 1941. "The vis cogitativa and Evaluation.” The New Scholasticism 15: 195-221.

Aristóteles. 1987. Acerca de la generación y la corrupción. Tratados breves de historia natural, edited by E. La Croce, A. Bernabé Pajares. Madrid: Gredos.

Barbado, M. 1946. Estudios de psicología experimental. Madrid: Consejo Superior de Investigaciones Científicas.

Byers, S. C. 2016. “Augustine's debt to Stoicism in the Confessions.” In The Routledge Handbook of the Stoic Tradition, edited by J. Sellars, : 56-69. Oxford-New York: Routledge.

Beuchot, M. 1994. "La teoría de las distinciones en la Edad Media y su influjo en la Edad Moderna.” Revista Española de Filosofía Medieval 1: 37-48.

Black, D. L. 2000. "Imagination and Estimation: Arabic Paradigms and Western Transformations.” Topoi 19/1: 59-75.

Blanco, C. 2014. Historia de la neurociencia. El conocimiento del cerebro y la mente desde una perspectiva interdisciplinar, Madrid: Biblioteca Nueva.

Castellote, S. 1963. “Antropología filosófica en la obra de Francisco de Valles.” Archivo Iberamericano de Historia de la Medicina y Antropología Médica 15: 77-120.

De Haan, D. D. 2014. "Moral Perception and the Function of the Vis Cogitativa in Thomas Aquinas's Doctrine of Antecedent and Consequent Passions." Documenti e studi sulla tradizione filosofica medievale 25: 289-330.

Edwards, M., 2012. "Suárez in a Late Scholastic Context. Anatomy, Psychology, and Authority.” In The Philosophy of Francisco Suárez, edited by B. Hill \& H. Lagerlund, 25-37. Oxford: Oxford University Press.

Fabro, C. 2008. Percezione e pensiero. Segni: EDIVI.

García Cuadrado, J. Á. 2015. "La armonía de facultades en la gnoseología de Francisco Suárez.” Pensamiento 71/267: 587-615.

Grabmann, M. 2006. “Naturaleza metodológica y actualidad de las «Disputaciones metafísicas» de Francisco Suárez.” Azafea 8: 149-179.

Jansen, R. 2005. Aquinas on the cogitative power and the generation of the sense appetite. Portland: The Catholic University of America.

Kemp, S. - Fletcher, G. J. O. 1993. “The Medieval Theory of the Inner Senses.” The American Journal of Psychology, 106/4: 559-576.

Knuuttila, S. 2014. “Suárez’s Psychology.”, In A Companion to Francisco Suárez, edited by V. Salas and R. Fastiggi, 192-220. Leiden-Boston: Brill. 
Lavin, I. 1992. "Memoria e senso di sé. Sul ruolo della memoria nella teoria della psicologia dall'antichità a Giambattista Vico.” In La cultura della memoria, edited by L. Bolzoni-P. Corsi, 291-317 . Bologna: Il Mulino.

Leijenhorst, C. 2007. "Cajetan and Suarez on Agent Sense. Metaphysics and Epistemology in Late Aristotelian Thought." In Forming the Mind. Essays on the Internal Senses and the Mind/Body Problem from Avicenna to the Medical Enlightenment, edited by H. Lagerlund, 237-262. Dordrecht: Kluwer.

Lombo, J. A. - Giménez Amaya, J. M. 2016. Biología y racionalidad: El carácter distintivo del cuerpo humano. Pamplona: EUNSA.

Madeira, J. 2009. "Francisco Valles Covarrubias: o galenismo renascentista depois de Andreas Vesalius.” Veritas (Porto Alegre) 54/3: 71-89.

Pereira, J. 2007. Suárez: Between Scholasticism and Modernity. Milwaukee (WI): Marquette University Press.

Piro, F. 2012. "E' sufficiente un solo senso interno? La psicologia dell'immaginazione nella prima età moderna e le sue difficoltà.” Lo Sguardo - Rivista di Filosofia 10/3:183-197.

South, J. 2001. “Francisco Suárez on Imagination.” Vivarium 39/1: 119-158.

Suárez, F. 1978-1991 De Anima (Commentaria una cum quaestionibus in libros de Aristotelis De anima), edited by S. Castellote. Madrid: Labor-Sociedad de Estudios y Publicación.

Summers, D. 1987. The Judgment of Sense. Renaissance Naturalism and the Rise of Aesthetics. Cambridge: Cambridge University Press.

Tellkamp, J. A. 2012. "Albert the Great on structure and function of the inner senses." In The Judeo-Christian-Islamic heritage. Philosophical and theological perspectives, edited by R. Taylor \& I. Omar, 305-324. Milwaukee (WI): Marquette University Press.

Tomás de Aquino. 1959. In Aristotelis librum De anima, edited by A. M. Pirotta. Turín-Roma: Marietti.

Vallés, F. 1556. Controversiarum medicarum et philosophicarum, Alcalá: J. Brocar. Vesalio, A. 1543. De humani corporis fabrica libri septem. Basilea: Ioannis Oporini.

Wolfson, H. A. 1935. "The Internal Senses in Latin, Arabic, and Hebrew Philosophic Texts.” The Harvard Theological Review 28/2: 69-133. 